\title{
Acidentes de trabalho entre profissionais da limpeza hospitalar em uma capital do Nordeste, Brasil
}

\author{
Workplace accidents among hospital cleaning professionals \\ at a hospital in the city of Maranhão, Brazil
}

Sâmea Cristina Santos Gomes (https://orcid.org/0000-0002-8503-6824) ${ }^{1}$

Isabela Vieira dos Santos Mendonça (https://orcid.org/0000-0001-8362-2427) ${ }^{2}$

Luana Pontes Oliveira (https://orcid.org/0000-0002-5340-8777) ${ }^{3}$

Arlene de Jesus Mendes Caldas (https://orcid.org/0000-0001-7087-8781) ${ }^{4}$
${ }^{1}$ Programa de PósGraduação em Saúde Coletiva, Universidade Federal do Maranhão (UFMA). Av. dos Portugueses 1966, Vila Bacanga. 65080-805 São Luís MA Brasil. cris_ samea@hotmail.com ${ }^{2}$ Departamento Acadêmico de Biologia, Instituto

Federal de Educação, Ciência e Tecnologia do Maranhão - IFMA. São Luís MA Brasil.

${ }^{3}$ Programa de Pós-

Graduação em Enfermagem,

UFMA. São Luís MA

Brasil.

${ }^{4}$ Departamento de

Enfermagem, UFMA. São

Luís MA Brasil.

\begin{abstract}
The goal of this study was to investigate the prevalence and factors associated with workplace accidents among hospital cleaning professionals. This is a cross-sectional, analytical study of a sample of 199 cleaning workers at six hospitals in São Luís, Maranhão, Brazil. We first ran a univariate analysis, and later the Poisson regression analyzes with robust variance were used, with hierarchized modeling of the data in order to estimate the prevalence ratios (PR) between the independent variables and the outcome (workplace accidents). The prevalence of workplace accidents is $13.57 \%, 81.48 \%$ of them with sharps. At the end of the multivariate analysis the following were found to be associated with workplace accidents: age between 18 and 30, not having a high-school degree, on the job for 2 to 5 years, inadequate segregation of health service waste (HSW), training only on admission, failure to consider the health hazards of hospital waste and failure to use personal protective equipment (PPE). Our findings reinforce the importance of continued health education, stressing periodic training and the use of PPE.

Key words Occupational health, Hospital housekeeping, Workplace accidents, Waste health services
\end{abstract}

Resumo O objetivo deste estudo foi investigar a prevalência e os fatores associados aos acidentes de trabalho entre profissionais da limpeza hospitalar. Foram entrevistados 199 trabalhadores da limpeza de seis hospitais em São Luís, Maranhão, Brasil. Primeiramente, realizou-se análise univariada e, posteriormente, utilizaram-se as análises de regressão de Poisson com variância robusta, com modelagem hierarquizada dos dados a fim de estimar razões de prevalências $(R P)$ entre as variáveis independentes e o desfecho (acidentes de trabalho). Houve prevalência de $13,57 \%$ de acidentes de trabalho, destes, $81,48 \%$ ocorridos com materiais perfurocortantes. Ao final da análise multivariada, identificaram-se fatores associados á ocorrência de acidentes de trabalho: idade de 18 a 30 anos, ter ensino médio incompleto, tempo de serviço de 2 a 5 anos, segregação inadequada dos resíduos de serviços de saúde (RSS), ter capacitação somente na admissão, não considerar os riscos dos resíduos à saúde e o não uso de equipamento de proteção individual (EPI). Os fatores avaliados apresentaram-se associados significativamente aos acidentes de trabalho e ressaltam a importância da educação permanente em saúde, com ênfase em capacitações periódicas e uso de EPI.

Palavras-chave Saúde do trabalhador, Serviço de limpeza hospitalar, Acidentes de trabalho, Resíduos de serviços de saúde 


\section{Introdução}

Os acidentes de trabalho com exposição a material biológico representam um grande desafio às instituições de saúde, pois se constituem como problema de saúde pública, sendo considerado o maior agravo à saúde do trabalhador ${ }^{1}$. De acordo com o Projeto Risco Biológico, do Sistema de Vigilância de Acidentes de Trabalho com Material Biológico em serviços de saúde brasileiros, de março de 2002 a novembro de 2016 foram registrados 14.078 acidentes de trabalho ${ }^{2}$.

No âmbito dos serviços de saúde, a exposição ao risco de infecção com material biológico não é restrita a um grupo de profissionais, pelo contrário, todos os trabalhadores estão expostos aos riscos. Os trabalhadores do setor saúde têm um risco 1,5 vezes maior de ter acidente de trabalho ou doença profissional comparativamente aos trabalhadores de outros setores ${ }^{3}$.

Entre estes trabalhadores, o trabalhador da limpeza e conservação dos serviços de saúde (TLCSS), em sua rotina de trabalho, manuseia materiais potencialmente infectantes, e, tratando-se de um ambiente hospitalar, o manejo desses materiais pode resultar em acidente de trabalho $\mathrm{o}^{4,5}$.

Os TLCSS podem acidentar-se em consequência das ações de outros profissionais da área da saúde que, ao descartarem de forma inadequada os materiais utilizados em procedimentos hospitalares, facilitam a ocorrência de acidentes ${ }^{6}$. Estudos realizados com estes trabalhadores os colocam em $2^{\circ}$ e $3^{\circ}$ lugares em número de acidentes ocorridos, sendo os resíduos perfurocortantes, os objetos mais envolvidos nesses acidentes ${ }^{7-9}$.

$\mathrm{Na}$ Índia $^{10}$ e no Irã ${ }^{11}$, estudos evidenciaram que um fator de risco comum para essses trabalhadores é o manejo dos perfurocortantes. Além disso, ainda existe a necessidade de efetiva prevenção dos acidentes com estes dispositivos, e estratégias que melhorem as condições de trabalho são extremamente necessárias.

No Brasil, em 2013, em estudo realizado em um hospital de Santa Maria (Rio Grande do Sul), com 157 trabalhadores do serviço de limpeza, foi estimada uma taxa de prevalência de $17,8 \%$ de acidentes de trabalho. Embora este estudo não tenha sido capaz de identificar fatores significativamente associados aos acidentes de trabalho, as porcentagens relatadas foram úteis para as ações permanentes de educação em saúde ${ }^{12}$.

Estudos descritivos tem sugerido que o déficit de capacitação, ausência ou inadequação do uso de equipamentos de proteção individual (EPI) e práticas inadvertidas do uso e descarte de per- furocortantes podem ser fatores relacionados à ocorrência de acidentes de trabalho ${ }^{7-9,13}$.

Nesse contexto, para entender quais os fatores associados à ocorrência dos acidentes de trabalho estão presentes na população estudada, utilizou-se a análise hierarquizada, considerando que a ocorrência de acidentes de trabalho pode ter influência direta ou indireta de determinados fatores, que vão desde os níveis proximais, até os intermediários e distais.

Frente à necessidade de aprofundar o conhecimento acerca da identificação dos fatores associados ao padrão de ocorrência desse evento, levando em consideração a realidade de acidentes nesta categoria, o presente trabalho teve como objetivo investigar a prevalência e os fatores associados aos acidentes de trabalho entre trabalhadores da limpeza e conservação (TLCSS) de hospitais no município de São Luís, Maranhão, Brasil.

\section{Métodos}

Trata-se de um estudo transversal do tipo analítico, desenvolvido no período de novembro de 2012 a março de 2015, no município de São LuísMA, em hospitais públicos e privados.

Optou-se por trabalhar com hospitais não especializados de médio e grande porte no município de São Luís-MA, por serem considerados os maiores geradores de Resíduos de Serviços de Saúde (RSS). Considerou-se hospital de médio porte aquele com capacidade de 50 a 149 leitos, e hospital de grande porte, com 150 a 499 leitos $^{14}$. Do total de 22 hospitais de São Luís-MA, nove $(40,9 \%)$ eram de médio e grande portes não especializados; destes, seis aceitaram participar do estudo, sendo quatro instituições públicas e duas privadas.

A população foi constituída pelos trabalhadores da limpeza e conservação dos hospitais em estudo. O cálculo amostral foi realizado considerando a população de 336 trabalhadores do serviço de limpeza com um erro amostral de 5 , sendo que 183 (54\% do total) fariam parte do conjunto amostral. Prevendo a possibilidade de perdas, acrescentou-se $10 \%$ a esse valor, chegando a uma amostra mínima de 199 trabalhadores.

Para a coleta de dados, inicialmente foram contactados os responsáveis (diretor) de cada hospital e solicitada a permissão para a realização do estudo. Para a instituição que concordou com a realização do estudo foi agendada uma reunião com serviços de Higienização e Limpeza. Nesta 
reunião, foi apresentada a proposta da pesquisa bem como a definição do cronograma para as atividades previstas durante a coleta de dados e a solicitação da relação dos trabalhadores da limpeza e conservação de cada instituição de saúde.

Cada trabalhador recebeu informações detalhadas sobre os objetivos do estudo, e, aos que concordaram em participar da pesquisa, foi solicitada a assinatura do Termo de Consentimento Livre e Esclarecido (TCLE).

Utilizou-se um formulário semiestruturado para coleta de dados, constituído com as seguintes variáveis distribuídas em três níveis: nível distal (sexo, idade em anos, escolaridade, tempo de serviço); nível intermediário (questões relacionadas com o conhecimento sobre o manejo dos RSS (definição, classificação, etapas do manejo e legislação específica); nível proximal (capacitação sobre o manejo dos RSS (sim/não; periodicidade), utilização de Equipamento de Proteção Individual-EPI ( $\operatorname{sim} /$ não; tipo) e ocorrência de acidentes de trabalho ( $\operatorname{sim} /$ não; tipo). O formulário foi preenchido por entrevistadores previamente treinados.

Considerou-se como acidentes de trabalho, todo incidente ocorrido com resíduos de serviços de saúde durante a prática laboral dos TLCSS no hospital em que se encontrava contratado no momento da pesquisa ${ }^{15}$.

A idade do trabalhador foi categorizada em 18-30 anos, 31-40 anos e > 40 anos; Sexo categorizado em masculino e feminino; Para escolaridade, considerou-se as categorias ensino médio completo e ensino médio incompleto; $\mathrm{O}$ tempo de serviço foi organizado em $<2$ anos, 2-5 anos, 6-10 anos, >10 anos; Definição, classificação, segregação, legislação, destinação e tratamento final dos RSS foram categorizadas em sim e não; Capacitação sobre o manejo dos RSS foi categorizada em mensal, bimestral/semestral, na admissão, sem capacitação; Riscos dos RSS à saúde foi categorizada em sim e não; Uso do EPI, sim e não.

Os dados foram analisados no programa Stata (StataCorp.,CollegeStation, Estados Unidos), versão 12 para Windows de domínio público. Primeiramente, foi realizada análise não ajustada onde foi testada a associação de todas as variáveis em relação ao acidente de trabalho. Foram mantidas para segunda fase aquelas que apresentaram p-valor $<0,20$. Para a segunda fase, utilizaram-se as análises de regressão de Poisson com variância robusta, com modelagem hierarquizada dos dados a fim de estimar razões de prevalências (RP) entre as variáveis independentes e o desfecho ${ }^{16}$. A análise do tipo hierarquizada propõe a classi- ficação das variáveis segundo sua influência no desfecho, classificando-as em distais, intermediárias e proximais, de acordo com o modelo teórico (Figura 1). As estimativas de associação foram ajustadas para as variáveis do mesmo nível hierárquico e dos níveis anteriores, possibilitando a permanência daquelas mais intensamente associadas ao desfecho de interesse ${ }^{17}$.

Foram incluídas de uma única vez, as variáveis do nível distal que na análise não ajustada apresentaram $\mathrm{p}<0,20$; permanecendo somente neste nível as variáveis que mantiveram $\mathrm{p}$-valor $\leq$ 0,05. Mantidas as variáveis do nível distal, o passo seguinte foi o estudo das variáveis do nível intermediário. Por fim, foram inseridas as variáveis do nível proximal de forma semelhante as dos níveis anteriores e permanecendo no modelo final as variáveis que mantiveram $\mathrm{p}$-valor $\leq 0,05$, estimados os valores das razões de prevalência (RP), tendo como categoria de referência $\mathrm{RP}=1$, construídos os intervalos de confiança de $95 \%$ e determinados os valores de $\mathrm{p}$.

Esse estudo é parte integrante da pesquisa intitulada "Avaliação do gerenciamento dos resíduos de serviços de saúde em São Luís-MA”, que foi apreciado e aprovado pelo Comitê de Ética em Pesquisa-CEP/HUUFMA.

\section{Resultados}

Dos 199 trabalhadores da limpeza que participaram do estudo, 13,57\% afirmaram ter se acidentado durante o seu processo de trabalho no último ano. Destes, $81,48 \%$ acidentaram-se por meio de objeto perfurocortante, $39,7 \%$ tinham 31 a 40 anos, $57,79 \%$ eram do sexo masculino, $85,43 \%$ com ensino médio completo, $44,7 \%$ tinham de 2 a 5 anos de tempo de serviço (Tabela 1).

Quanto aos conhecimentos sobre o manejo dos RSS, constatou-se que $82,23 \%$ dos trabalhadores desconhecem o que sejam os resíduos e como são classificados; 57,29\% desconhecem a existência da legislação especifica sobre RSS; 93,97\% dos trabalhadores afirmaram que a etapa de segregação é realizada, mas, 63,13\% disseram que desconhecem a existência do tratamento e da destinação final dos RSS (Tabela 1).

A capacitação sobre o manejo dos RSS foi referida por $82,92 \%$ dos TLCSS. Porém, quando se avaliou a periodicidade dessas capacitações, 47,74\% dos TLCSS mencionaram haver capacitação mensal e/ou semestral, e 23,12\% disseram ter ocorrido apenas na admissão. Os riscos com o manuseio dos RSS foram citados como existentes 


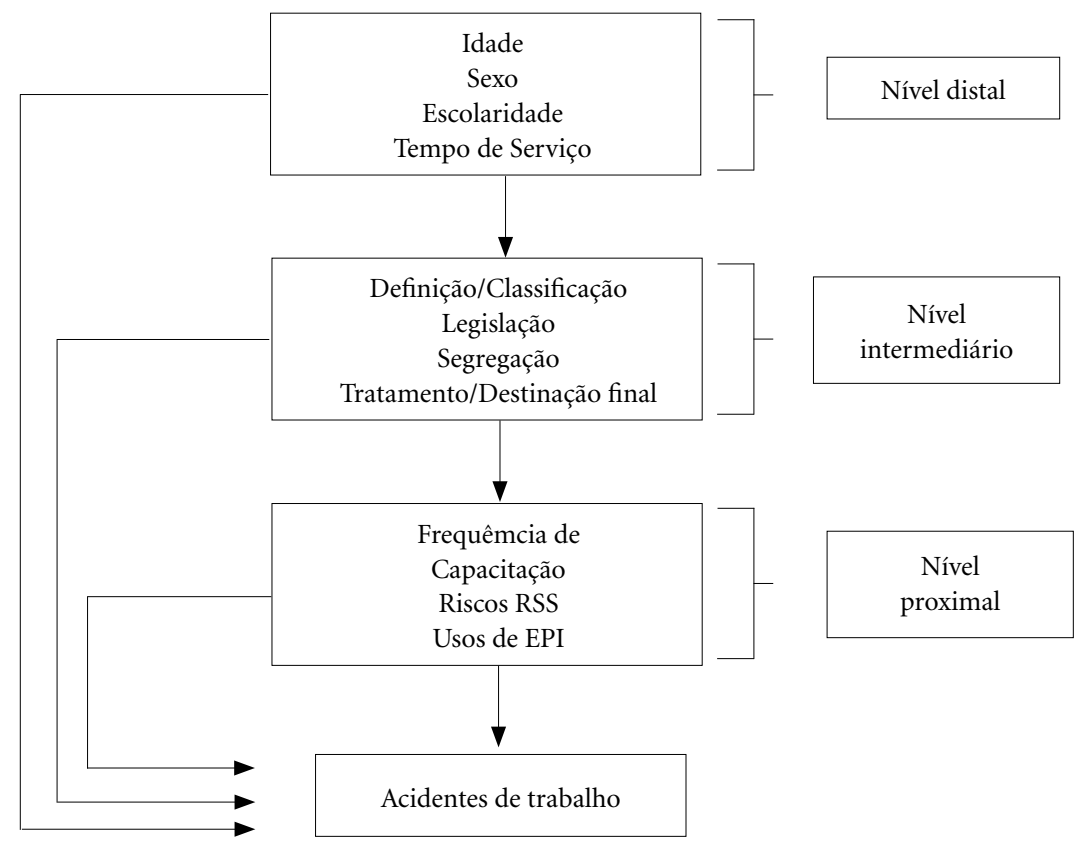

Figura 1. Fluxograma da relação hierárquica entre as variáveis e sua relação com o desfecho.

por $87,94 \%$ dos TLCSS. Quanto ao uso dos Equipamentos de Proteção Individual (EPI), 85,43\% informaram utilizar os equipamentos recomendados (essencialmente uso de botas, luvas e máscaras na sua rotina de trabalho) (Tabela 1 ).

$\mathrm{Na}$ análise não ajustada, a faixa etária de 18 a 30 anos $(\mathrm{RP}=1,15 ; \mathrm{p}<0,001)$, ter ensino médio incompleto $(\mathrm{RP}=2,88$; $\mathrm{p}<0,001)$ e capacitação somente na admissão $(\mathrm{RP}=4,15 ; \mathrm{p}<0,001)$ apresentaram-se associados à ocorrência de acidentes de trabalho (Tabela 1).

$\mathrm{Na}$ análise ajustada, em que se utilizou a análise hierarquizada, dentre as variáveis distais, a idade de 31 a 40 anos $(R P=0,18 ; p<0,001)$, e o tempo de serviço de 6 a 10 anos $(\mathrm{RP}=0,09 ; \mathrm{p}$ $=0,004)$ associaram-se como fator de proteção a ocorrência de acidente de trabalho. Por outro lado, a faixa etária de 18 a 30 anos (RP: 1,16; p < 0,001) apresentou-se associada aos acidentes de trabalho. Ademais, os trabalhadores com ensino médio incompleto quase triplicam a chance de se acidentarem no trabalho $(\mathrm{RP}=2,98 ; \mathrm{p}<0,001)$ e o tempo de serviço de 2 a 5 anos $(\mathrm{RP}=1,16$; $\mathrm{p}<$ $0,001)$ tem cerca de $16 \%$ de prevalência aumentada para a ocorrência de acidentes de trabalho (Tabela 2).
A segregação inadequada dos RSS $(\mathrm{RP}=2,31$; $\mathrm{p}=0,009$ ) foi a única variável do nível intermediário que mostrou associação significativa com a ocorrência de acidentes de trabalho (Tabela 2).

No modelo final da análise hierarquizada as variáveis que mantiveram associação com a ocorrência de acidentes de trabalho foram: idade de 18 a 30 anos $(\mathrm{RP}=1,16 ; \mathrm{p}<0,001)$, ter ensino médio incompleto $(\mathrm{RP}=2,98 ; \mathrm{p}<0,001)$, tempo de serviço de 2 a 5 anos $(R P=1,16 ; p<0,001)$, segregação inadequada dos $\mathrm{RSS}(\mathrm{RP}=2,31 ; \mathrm{p}=$ $0,009)$, capacitação somente na admissão ( $R P=$ 2,63; p < 0,001), não considerar os riscos dos RSS à saúde $(\mathrm{RP}=1,61 ; \mathrm{p}=0,002)$ e não uso de EPI $(R P=5,10 ; p=0,005)$. A idade de 31 a 40 anos e o tempo de serviço de 6 a 10 anos mostraram associação protetora com a ocorrência de acidente de trabalho (Tabela 2).

\section{Discussão}

Com base em uma revisão da literatura nacional e internacional, verifica-se que há uma consistência nos resultados encontrados neste estudo. Isto tanto para a prevalência quanto para os fatores 
Tabela 1. Análise não ajustada das características dos trabalhadores da limpeza e conservação, em relação aos acidentes de trabalho ocorridos nos hospitais públicos e privados. São Luís-MA, 2015.

\begin{tabular}{|c|c|c|c|c|c|c|c|}
\hline \multirow{3}{*}{ Variáveis } & \multicolumn{7}{|c|}{ Acidentes de Trabalho } \\
\hline & \multirow{2}{*}{$\mathbf{N}$} & \multirow{2}{*}{$\%$} & \multirow{2}{*}{$\begin{array}{c}\text { Não } \\
\mathrm{n}=172(\%)\end{array}$} & \multirow{2}{*}{$\begin{array}{c}\text { Sim } \\
\mathbf{n}=27(\%)\end{array}$} & \multicolumn{3}{|c|}{ Análise não ajustada } \\
\hline & & & & & $\mathbf{R P}$ & IC 95\% & p-valor \\
\hline \multicolumn{8}{|l|}{ Variáveis distais } \\
\hline \multicolumn{8}{|l|}{ Idade (anos) } \\
\hline $18-30$ & 77 & 38,69 & $52(30,23)$ & $25(92,60)$ & 1,15 & $1,02-1,21$ & $<0,001^{\star}$ \\
\hline $31-40$ & 79 & 39,70 & $78(45,34)$ & $1(3,70)$ & 0,15 & $0,08-0,42$ & $0,005^{\star}$ \\
\hline$>40$ & 43 & 21,61 & $42(24,41)$ & $1(3,70)$ & 1 & & \\
\hline \multicolumn{8}{|l|}{ Sexo } \\
\hline Masculino & 115 & 57,79 & $97(56,40)$ & $18(66.67)$ & 1,46 & $0,68-3,09$ & 0,323 \\
\hline Feminino & 84 & 42,21 & $75(43,60)$ & $9(33,33)$ & 1 & & \\
\hline \multicolumn{8}{|l|}{ Escolaridade } \\
\hline Ensino Médio Incompleto & 29 & 14,57 & $59(34,30)$ & $22(81,48)$ & 2,88 & $1,82-4,78$ & $<0,001^{\star}$ \\
\hline Ensino Médio Completo & 170 & 85,43 & $113(65,70)$ & $5(18,52)$ & 1 & & \\
\hline \multicolumn{8}{|l|}{ Tempo de Serviço (anos) } \\
\hline$<2$ & 83 & 41,70 & $76(44,18)$ & $7(25,92)$ & 1,58 & $1,72-4,76$ & $0,002^{*}$ \\
\hline $2-5$ & 89 & 44,70 & $79(45,93)$ & $10(37,03)$ & 1,79 & $1,98-4,37$ & $0,004^{*}$ \\
\hline $6-10$ & 19 & 9,58 & $10(5,81)$ & $9(33,33)$ & 0,87 & $0,12-0,98$ & $0,034^{*}$ \\
\hline$>10$ & 8 & 4,02 & $7(4,08)$ & $1(3,72)$ & 1 & & \\
\hline \multicolumn{8}{|l|}{ Variáveis intermediárias } \\
\hline \multicolumn{8}{|l|}{ Definição/Classificação RSS ${ }^{* *}$} \\
\hline Sim & 35 & 17,77 & $34(19,76)$ & $1(3,71)$ & 1 & & \\
\hline Não & 162 & 82,23 & $136(80,24)$ & $26(96,29)$ & 5,61 & $0,78-40,2$ & $0,086^{*}$ \\
\hline \multicolumn{8}{|l|}{ Legislação RSS } \\
\hline $\operatorname{Sim}$ & 85 & 42,71 & $70(40,70)$ & $15(55,55)$ & 1 & & \\
\hline Não & 114 & 57,29 & $102(59,30)$ & $12(44,45)$ & 1,23 & $0,81-40,2$ & 0,320 \\
\hline \multicolumn{8}{|l|}{ Segregação RSS } \\
\hline Sim & 187 & 93,97 & $164(95,34)$ & $23(85,18)$ & 1 & & \\
\hline Não & 12 & 6,03 & $8(4,66)$ & $4(14,82)$ & 2,71 & $1,11-6,59$ & $0,028^{\star}$ \\
\hline \multicolumn{8}{|c|}{ Tratamento e Destinação Final RSS } \\
\hline Sim & 73 & 36,87 & $64(37,20)$ & $10(37,03)$ & 1 & & \\
\hline Não & 125 & 63,13 & $108(62,80)$ & $17(62,97)$ & 0,99 & $0,47-2,05$ & 0,984 \\
\hline \multicolumn{8}{|l|}{ Variáveis proximais } \\
\hline \multicolumn{8}{|l|}{ Capacitação RSS } \\
\hline Na admissão & 46 & 23,12 & $31(18,03)$ & $15(55,55)$ & 4,15 & $2,09-8,25$ & $<0,001^{\star}$ \\
\hline Anual & 24 & 12,06 & $19(11,04)$ & $5(18,52)$ & 1,65 & $0,69-3,97$ & 0,258 \\
\hline Mensal/Semestral & 95 & 47,74 & $89(51,74)$ & $6(22,22)$ & 1 & & \\
\hline Sem Capacitação & 34 & 17.09 & $33(19,19)$ & $1(3,71)$ & 1,18 & $0,02-1,33$ & 0,395 \\
\hline \multicolumn{8}{|l|}{ Riscos RSS } \\
\hline Sim & 175 & 87,94 & $158(91,86)$ & $17(62,96)$ & 1 & & \\
\hline Não & 24 & 12,06 & $14(8,14)$ & $10(37,04)$ & 1,59 & $1,38-2,91$ & $0,019^{*}$ \\
\hline \multicolumn{8}{|l|}{$\mathrm{EPI}^{\star \star \star *}$} \\
\hline Sim & 170 & 85,43 & $164(95,34)$ & $6(22,22)$ & 1 & & \\
\hline \multirow[t]{2}{*}{ Não } & 29 & 14,57 & $8(4,66)$ & $21(77,78)$ & 4,28 & $1,76-28,8$ & $0,005^{\star}$ \\
\hline & 199 & 100,0 & $172(100,0)$ & $27(100,0)$ & & & \\
\hline
\end{tabular}

*Variáveis com p-valor $<0,20{ }^{* *}$ RSS= Resíduos de Serviços de Saúde ${ }^{* * *}$ EPI = Equipamento de Proteção Individual RP: Razão de Prevalência; IC: Intervalo de Confiança; p: valor de p calculado a partir do Modelo de Regressão de Poisson com variância robusta.

associados à ocorrência de acidentes de trabalho. Fatores estes analisados com base estatística, utilizando modelo de regressão com modelagem hierarquizada. 
Tabela 2. Análise hierarquizada ajustada por regressão logística múltipla dos fatores associados aos acidentes de trabalho. São Luís, Maranhão, Brasil, 2015.

\begin{tabular}{lccc}
\hline \multicolumn{1}{c}{ Variáveis } & RP & IC 95\% & p-valor \\
\hline Idade de 18 a 30 anos & 1,16 & $1,02-1,27$ & $<0,001$ \\
Idade de 31 a 40 anos & 0,18 & $0,03-0,93$ & $<0,001$ \\
Ensino médio incompleto & 2,98 & $1,87-4,75$ & $<0,001$ \\
Tempo de serviço de 2 a 5 anos & 1,16 & $1,06-1,34$ & $<0,001$ \\
Tempo de serviço de 6 a 10 anos & 0,09 & $0,06-0,31$ & 0,004 \\
Segregação inadequada dos RSS & 2,31 & $1,02-4,96$ & 0,009 \\
Capacitação na admissão & 2,63 & $1,38-4,99$ & $<0,001$ \\
Riscos de RSS à saúde & 1,61 & $1,45-5,83$ & 0,002 \\
Não uso de EPI** & 5,10 & $1,62-15,90$ & 0,005 \\
\hline
\end{tabular}

${ }^{\star}$ RSS= Resíduos de Serviços de Saúde; ${ }^{\star * E P I ~=~ E q u i p a m e n t o ~ d e ~ P r o t e c ̧ a ̃ o ~ I n d i v i d u a l ; ~ R P: ~ R a z a ̃ o ~ d e ~ P r e v a l e ̂ n c i a ; ~ I C: ~ I n t e r v a l o ~ d e ~}$ Confiança; p: valor de p calculado a partir do Modelo de Regressão de Poisson com variância robusta.

Em relação à prevalência, o presente estudo apresentou uma taxa de $13,57 \%$ de acidentes de trabalho entre os trabalhadores da limpeza e conservação dos seis hospitais estudados. Estudos recentes relatam taxas de acidentes variando de $6,5 \%^{7}, 11,6 \%{ }^{5}$ a $22,8 \%{ }^{11}$. Tais prevalências parecem divergir devido às metodologias utilizadas no desenvolvimento dos estudos (ora desenhos metodológicos com dados primários ${ }^{7}$ ora com dados secundários ${ }^{5}$ ), pela observação do evento em um único estabelecimento hospitalar ${ }^{7,12}$, pelo número de participantes envolvidos ${ }^{5,12}$ e pela subnotificação dos acidentes de trabalho ${ }^{5}$.

A análise hierarquizada mostrou associação estatisticamente significante com a idade, escolaridade, tempo de serviço, segregação inadequada dos RSS, capacitação sobre o manejo dos RSS na admissão, riscos dos RSS à saúde e o não uso do EPI.

No modelo inicial, que incluía variáveis consideradas distais ao desfecho, a análise aponta para uma população constituída por adultos jovens, de baixa escolaridade e com tempo de serviço menor que cinco anos. $\mathrm{O}$ ensino médio incompleto e o tempo de serviço de 2 a 5 anos mostraram-se associados aos acidentes de trabalho. A escolaridade é um fator que deve ser considerado e levado à reflexão, pois os índices de acidentes de trabalho são altos nessa população e o manuseio dos RSS requer uma compreensão adequada sobre os riscos aos quais estão submetidos.

As variáveis, idade de 31 a 40 anos, e o tempo de serviço de 6 a 10 anos apresentam-se como fatores de proteção à ocorrência de acidente de trabalho. Estes achados sugerem que quanto maior a idade e o tempo de serviço maior familiarização do trabalhador com o manejo dos RSS e consequentemente menor ocorrência de acidentes.

É importante salientar que a idade de 18 a 30 anos corrobora achados da literatura e da prática em que a frequência de acidentes de trabalho tem sido maior nesta faixa etária ${ }^{2,3,16}$. Neste estudo, 25 dos 27 acidentes relatados ocorreram com trabalhadores nesta faixa de idade, o que pode ser justificado pela inserção desses trabalhadores no mercado de trabalho de forma precoce, de maneira terceirizada e sem treinamentos adequa$\operatorname{dos}^{18}$. Ademais, tais acidentes nessa faixa etária podem também estar relacionados com o tipo de tarefa que o trabalhador estava realizando no momento do acidente.

Convém ressaltar que os trabalhadores da limpeza pesquisados são contratados por empresas terceirizadas, e nos últimos anos, observa-se a necessidade de se discutir sobre a segurança do trabalho nas empresas de serviços terceirizados, uma vez que, pela natureza do trabalho e multiplicidade dos locais ocupacionais, existe grande dificuldade de controlar os riscos à saúde e a integridade física dos trabalhadores ${ }^{19}$, o que pode levar a potenciais acidentes de trabalho ${ }^{20}$.

Outro achado apontado pela análise hierarquizada como fator associado aos acidentes de trabalho foi a segregação inadequada dos RSS. Embora as variáveis de conhecimentos sobre o manejo dos RSS, como a definição, classificação e legislação não tenham apresentado associação significativa com a ocorrência de acidente de trabalho, outros estudos ${ }^{10,19-21}$ apontam que um insuficiente conhecimento a respeito desses itens, assim como o desconhecimento sobre o tratamento e a destinação final dos mesmos pode contribuir para uma segregação inadequada. 
Encontramos também no presente estudo que 23 dos 27 acidentes relatados ocorreram com aqueles trabalhadores que afirmaram conhecer a etapa de segregação dos RSS. Este achado parece sugerir que conhecer a etapa de segregação não necessariamente garante a realização desta etapa adequadamente.

As três variáveis consideradas proximais (capacitação sobre os RSS, riscos dos RSS e uso de EPI), mostraram-se associadas à ocorrência de acidentes de trabalho. A capacitação sobre os RSS foi relatada como presente pela maioria dos trabalhadores. No entanto, quando se verifica a frequência de capacitações sobre o manejo dos RSS, os achados apontam que $32,61 \%$ dos trabalhadores que sofreram acidente de trabalho relataram a realização de capacitação apenas no momento da admissão. Este resultado parece ter relação com a rotatividade dos trabalhadores nas empresas, visto que o trabalho é terceirizado e muitas vezes o trabalhador só chega a realizar a capacitação no momento da contratação.

Considera-se que a educação permanente é uma ferramenta fundamental para obtenção de melhores resultados ${ }^{22}$ e o trabalho de capacitação constante ${ }^{12}$, aliado ao número de horas destinadas às capacitações dos trabalhadores também contribui para a diminuição dos acidentes ${ }^{23}$.

A implementação da educação permanente faz-se necessária também devido ao fato de que dentre os acidentes relatados, 81,48\% tenham ocorrido com material perfurocortante, o que re-

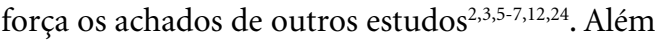
disso, alguns trabalhadores que sofreram acidentes consideraram não haver riscos para a sua saúde o manuseio dos RSS.

O risco no manuseio dos RSS está, principalmente, vinculado aos acidentes que ocorrem devido às falhas com o descarte de materiais perfurocortantes sem utilização de proteção mecânica, ocasionando grande possiblidade de transmissão de doenças ${ }^{8}$. Soma-se a isso, que este grupo de trabalhadores muitas vezes realiza a atividade de limpeza pela primeira vez, sem capacitações direcionadas à prática de suas atividades diárias ou com tempo de capacitação reduzido (no caso, capacitação somente no momento da admissão), o que contribui, por exemplo, para as dificuldades no uso de $\mathrm{EPI}^{12}$.

Destaca-se que, embora o uso de EPI no ambiente de trabalho tenha sido relatado pelos trabalhadores, e as luvas, botas e máscaras tenham sido os mais utilizados $(33,67 \%)$, a chance de acidente de trabalho entre aqueles que não utilizam o EPI é cinco vezes maior segundo a análise rea- lizada. Dos trabalhadores que sofreram acidentes, $57,14 \%$ utilizam com frequência apenas a máscara como EPI. Semelhante a nosso estudo, Paraíso e Gouveia ${ }^{12}$ evidenciaram em seu estudo que $32 \%$ dos trabalhadores pesquisados e que sofreram acidentes relataram não usar EPI adequadamente.

Vale ressaltar que o uso exclusivo de EPI não é suficiente para garantir total segurança, porém pode contribuir para a adoção de práticas seguras e minimização de riscos ${ }^{3,7,12}$.

O uso de EPI, a manutenção de programas de educação permanente, mudanças no comportamento profissional e a adoção de medidas são apontados como fatores preventivos na maioria dos trabalhos ${ }^{3,11,25}$.

Mesmo levando em consideração a robustez dos resultados encontrados neste estudo, cabe ressaltar algumas limitações que podem contribuir para comprometer a consistência dos resultados encontrados: 1) Tamanho da população: o estudo ter sido realizado somente em seis hospitais do município de São Luís-MA e não ter utilizado a totalidade de trabalhadores existentes nesses estabelecimentos de saúde; 2) a concentração de questões relacionadas ao manejo dos RSS, questões mais específicas como as características laborais (jornada de trabalho, setor de trabalho, turno de trabalho, outro emprego, horas extras, carga horária semanal no hospital pesquisado), poderiam ampliar a consistência dos dados. Porém, cabe ressaltar que mesmo com esta limitação, as questões utilizadas trouxeram um enfoque diferenciado ao estudo, pois elencaram o manejo dos RSS no setor e como este é conhecido e trabalhado; 3 ) a questão sobre a ocorrência de acidentes de trabalho baseou-se em lembranças (viés de memória).

O estudo apresenta pontos fortes, ao evidenciar que a exposição ocupacional entre os TLCSS ainda se mostra como um desafio, principalmente quando relacionada ao manejo dos RSS no ambiente hospitalar, visto que nesse cenário os trabalhadores estão expostos a vários riscos ocupacionais. Além disso, a relevância do estudo para o grupo estudado contribui para a busca de alternativas que possam minimizar os impactos negativos dos acidentes na vida dos trabalhadores, destacando alguns elementos que merecem maior atenção nas ações de educação no serviço implementadas com o grupo em estudo.

Conclui-se que a identificação da prevalência e dos fatores associados aos acidentes de trabalho entre trabalhadores da limpeza e conservação pode permitir intervenções sobre comportamentos de risco e melhoria na qualidade das condi- 
ções de trabalho. Além disso, o estudo ressalta a importância da educação permanente em saúde, para diminuição do risco de acidentes, com ênfase na realização de capacitações periódicas e o uso dos equipamentos de proteção individual rotineiramente.
A ocorrência dos acidentes de trabalho entre os TLCSS é um importante problema de saúde pública que ainda demanda de estudos epidemiológicos no Brasil, não sendo tarefa fácil observar à ocorrência do desfecho nessa população.

\section{Colaboradores}

Os autores SCS Gomes e AJM Caldas participaram da concepção, planejamento do estudo, análise de dados e interpretação e redação do manuscrito. Os autores IVS Mendonça e LP Oliveira participaram da interpretação, redação final e revisão crítica. Todos os autores aprovaram a versão final do manuscrito encaminhada. 


\section{Referências}

1. Zangirolani LTO, Cordeiro M, Medeiros MAT, Stephan C. Topologia do risco de acidentes do trabalho em Piracicaba, SP. Rev Saude Publica 2008; 42(2):287-293.

2. Projeto Risco Biológico (PSBIO). Sistema de vigilância de acidente de trabalho com material biológico em serviços de saúde. Projeto Risco Biológico. Relatório PSBIO 2016. [acessado 2017 Abr 10]. Disponível em: http://www.riscobiologico.org/psbio/psbio_201611. pdf

3. Martins MDS. Epidemiologia dos Acidentes de Trabalho em Instituições Públicas de Saúde - Fatores Associados e Repercussões [tese]. Porto: Universidade do Porto; 2014.

4. Ream PSF, Tipple AFV, Salgado TA, Sousa ACS, Souza SMB, Galdino-Junior H, Alves SB. Hospital housekeepers: Victims of ineffective hospital waste management. Arch Environ Occup Health 2015; 11:1-8.

5. Ream PSF, Tipple AFV, Barros DX, Souza ACS, Pereira MS. Biological risk among hospital housekeepers. Arch Environ Occup Health 2016; 71(2):59-65.

6. Murofuse NT, Marziale MHP, Gemelli LMG. Acidente com material biológico em hospital universitário do oeste do Paraná. Rev Gaúcha Enferm 2005; 26(2):168179.

7. Lima LM, Oliveira CC, Rodrigues KMR. Exposição ocupacional por material biológico no Hospital Santa Casa de Pelotas - 2004 a 2008. Esc Anna Nery 2011; 15(1):96-102.

8. Morais NO, Paniago AMM, Negri AC, Oliveira AO, Cunha VC, Oliveira SMV. Exposição ocupacional com material potencialmente contaminado entre profissionais da área de apoio. Cogitare Enferm 2009; 14(4):709-713.

9. Dias MAC, Machado AA, Santos BM. O. Acidentes ocupacionais com exposição a material biológico: retrato de uma realidade. Medicina 2012; 45(1):12-22.

10. Sharma A, Sharma V, Sharma S, Singh, P. Awareness of Biomedical Waste Management Among Health Care Personnel in Jaipur, India. OHDM 2013; 12(1):32-40.

11. Lakbala P, Azar FE, KamalI H. Needlestick and sharps injuries among housekeeping workers in hospitals of Shiraz, Iran. BMC Res Notes 2012; 5:276.

12. Ceron MDS. Serviço Hospitalar de Limpeza e Acidentes de Trabalho: contribuições da enfermagem [dissertação]. Santa Maria: Universidade Federal de Santa Maria; 2013

13. Mathur V, Dwivedi S, Hassan M, Misra R. Knowledge, attitude and practices about biomedical waste management among healthcare personnel: a cross-sectional study. Indian J Community Med 2011; 36(2):143-145

14. Brasil. Ministério da Saúde (MS). Portaria no 2.224, de 05 de dezembro de 2002. Estabelece o sistema de classificação hospitalar do Sistema Único de Saúde. Diário Oficial da União 2002; 6 dez.
15. Brasil. Ministério do Trabalho e do Emprego (TEM). Norma Regulamentadora no 32. Estabelece diretriz básica para a implementação de medidas de proteção à segurança e à saúde dos trabalhadores em serviço de saúde. Portaria n. ${ }^{\circ} 1.748$, de 30 de agosto de 2011. Diário Oficial da União 2011; 31 ago.

16. Hirakata VN. Estudos Transversais e Longitudinais com Desfechos Binários: qual a melhor medida de efeito a ser utilizada? Clin Biomed Res 2009; 29(2):174-176.

17. Fuchs SC, Victora CG, Fachel J. Modelo hierarquizado: uma proposta de modelagem aplicada à investigação de fatores de risco. Rev Saude Publica 1996; 30(2):168-178.

18. Cruz EDA, Pimenta FC, Hayashida M, Eidt M, Gir E. Detecção de Staphylococcus aureus na boca de trabalhadores da limpeza hospitalar. Rev Lat Am Enfermagem [Internet] 2011; 19(1). [acessado 2016 Set 10]. Available from: www.scielo.br/pdf/rlae/v19n1/pt_13. pdf

19. Pandovani A. Serviços terceirizados de limpeza e conservação: Aspectos gerais. 2009. [acessado 2016 Set 10]. Disponível em: http://www.areaseg.com/pdf/ sstemservicosterceirizados.pdf

20. Chillida MSP, Cocco, MIM. Saúde do trabalhador \& terceirização: perfil de trabalhadores de serviço de limpeza hospitalar. Rev Lat Am Enfermagem 2004; 12(2):271-276

21. Mochungong PIK. The plight of clinical waste pickers: evidence from the Northwest region of Cameroon. J Occup Health 2010; 52(2):142-145.

22. Al-Emad AA. Assessment of medical waste management in the main hospitals in Yemen. East Mediterr Health J 2011; 17(10):730-737.

23. Goetten LF. Sensibilização dos profissionais de saúde para redução de resíduos sólidos de serviços de saúde [dissertação]. Curitiba: Universidade Tecnológica Federal do Paraná; 2013.

24. Khalaf A-Sa. Assessment of Medical Waste Management in Jenin District Hospitals [dissertation]. Nablus: Faculty of Graduate Studies at An-Najah Nacional University; 2009.

25. Martins A, Coelho AC, Vieira M, Matos M, Pinto M. Age and years in practice as factors associated with needlestick and sharps injuries among health careworkers in a Portuguese hospital. Accid Anal Prev 2012; 47:11-15.

Artigo apresentado em 03/05/2017

Aprovado em 07/04/2018

Versão final apresentada em 09/04/2018 
